\title{
THE GRS-CONDITION AND SYMMETRY OF WEIGHTED $L^{1}$-ALGEBRAS
}

\author{
MICHAEL LEINERT \\ Institut für Angewandte Mathematik \\ Ruprecht-Karls-Universität Heidelberg \\ Im Neuenheimer Feld 294 \\ D-69120 Heidelberg, Germany \\ E-mail: leinert@math.uni-heidelberg.de
}

This note is about joint work with Gero Fendler and Karlheinz Gröchenig. Complete details will be given in [FGL]. Symmetry of Bannach $*$-algebras is of interest for several reasons, one of them being its relation to inverse-closedness in a bigger algebra. This for instance has concrete applications in signal analysis and numerical analysis (see [GL1], [GL2], [S]). In the applied context, weighted $L^{1}$-algebras are important, since the weight allows one to model a specific decay at infinity according to what one wants or needs.

Looking at the case of the trivial weight $\omega \equiv 1$ first, one sees that for groups with exponential growth the $L^{1}$-algebra "usually" is non-symmetric. For groups with polynomial growth, non-symmetry can happen, too (see [FRW]), but for compactly generated groups of polynomial growth, using his structure theorem and [Lu], Losert [Lo] has shown that the $L^{1}$-algebra is symmetric. So it seems reasonable to consider weighted $L^{1}$-algebras on such groups.

A locally compact group $G$ is called compactly generated if there is a compact neighbourhood $U$ of the identity $e$ with $G=\bigcup_{k=1}^{\infty} U^{k}$. We then call $U$ a generating neighbourhood. We say that a compactly generated group $G$ has (at most) polynomial growth if there is a generating neighbourhood $U$ and constants $C>0$ and $D \in \mathbb{N}$ such that $\left|U^{k}\right| \leq C k^{D}$ for all $k \in \mathbb{N}$. Here and in the following, $|U|$ denotes the Haar measure of $U$. The $L^{1}$ algebra of the group $G$ with the usual norm $\|f\|_{1}=\int|f(x)| d x$, where $d x$ denotes the Haar measure on $G$, and the usual *-algebra structure is denoted by $L^{1}(G)$. By a weight on $G$ we mean a locally bounded measurable function $\omega: G \rightarrow \mathbb{R}^{+}$satisfying $\omega(e) \geq 1$, where $e$ is the identity element of $G, \omega\left(x^{-1}\right)=\omega(x)$, and $\omega(x y) \leq \omega(x) \omega(y)$ for all $x, y \in G$. The

2000 Mathematics Subject Classification: 46-06, 43A20.

Key words and phrases: weighted $L^{1}$-algebra, symmetry, polynomial growth, GRS-condition, condition $(S)$. 
corresponding weighted $L^{1}$-algebra $L_{\omega}^{1}(G)=\left\{f \in L^{1}(G)\left|\int\right| f(x) \mid \omega(x) d x<\infty\right\}$ with norm $\|f\|_{1, \omega}=\int|f(x)| \omega(x) d x$ is an (actually dense) Banach *-subalgebra of $L^{1}(G)$. The weight $\omega$ is said to satisfy the Gelfand-Raikov-Shilov condition (GRS-condition) if $\omega\left(x^{n}\right)^{\frac{1}{n}} \rightarrow 1$ for every $x \in G$. We say that $\omega$ satisfies condition $(\mathbf{S})$ if $\sup _{y \in U^{n}} \omega(y)^{\frac{1}{n}} \rightarrow 1$ for some generating neighbourhood $U$ of $G$. Here $U^{n}=\left\{x_{1} x_{2} \ldots x_{n} \mid x_{1}, \ldots, x_{n} \in U\right\}$. If condition $(S)$ holds with respect to some generating neighbourhood $U$, it holds for every generating neighbourhood of $G$. Since every $x \in G$ is contained in some generating neighbourhood $U$, condition $(S)$ implies the GRS-condition. For explicit examples of weights satisfying these conditions see [FGLLM].

If $A$ is a Banach $*$-algebra, for $a \in A$ we denote by $\sigma_{A}(a)$ and $r_{A}(a)$ the spectrum and the spectral radius of $a$, respectively. $A$ is called symmetric, if $\sigma_{A}\left(a^{*} a\right) \subset[0, \infty)$ for all $a \in A$. This is equivalent to saying that $\sigma_{A}(a) \subset \mathbb{R}$ for all $a=a^{*} \in A$ (see [SF]).

THEOREM. Let $G$ be a locally compact, compactly generated group of polynomial growth and $\omega$ a weight on $G$. The following conditions are equivalent:

(i) $\omega$ satisfies the GRS-condition.

(ii) $\omega$ satisfies condition $(S)$.

(iii) $L_{\omega}^{1}(G)$ is symmetric.

(iv) $\sigma_{L_{\omega}^{1}(G)}(f)=\sigma_{L^{1}(G)}(f)$ for all $f \in L_{\omega}^{1}(G)$.

In [FGLLM], this was proved under the somewhat awkward technical assumption that the weight $\omega$ be "tempered". But this condition can be dispensed with, as we shall see.

Lemma 1. Let $\omega$ be a weight on $\mathbb{R}$. If $\omega(n)^{\frac{1}{n}} \rightarrow 0$, then $\omega$ satisfies condition $(S)$.

Proof. Assuming $\omega(n)^{\frac{1}{n}} \rightarrow 0$, from the fact that the logarithm of $\omega$ is subadditive, one can derive $\lim _{t \rightarrow \infty} t^{-1} \log \omega(t)=0$. To check condition $(S)$ using the generating neighbourhood $[-1,1]$, choose $x_{k} \in[0, k]$ such that $\left|\sup _{x \in[-k, k]} \omega(x)^{1 / k}-\omega\left(x_{k}\right)^{1 / k}\right|<\frac{1}{k}$. Since $1 \leq \lim \sup _{k \rightarrow \infty} \omega\left(x_{k}\right)^{\frac{1}{k}} \leq \limsup _{k \rightarrow \infty} \omega\left(x_{k}\right)^{\frac{1}{x_{k}}}=1$, we obtain that condition $(S)$ is satisfied.

LEMMA 2. If $\omega$ is a weight on a compactly generated locally compact abelian group $G$, condition $(S)$ and the GRS-condition are equivalent.

Proof. Since $G$ is of the form $\mathbb{R}^{d} \times \mathbb{Z}^{\ell} \times K$, where $K$ is a compact group, and since $\omega$ is dominated by the product of its restrictions to each coordinate, it suffices to consider weights on $\mathbb{R}, \mathbb{Z}$, and $K$. Assuming the GRS-conditon, we obtain condition $(S)$ on $\mathbb{R}$ by Lemma 1. A similar argument works for $\mathbb{Z}$. Since $\omega$ is bounded on $K$, condition $(S)$ holds on $K$, too.

If $G$ is a compactly generated locally compact group, from results of Losert [Lo] and Guivarc'h $[\mathrm{G}]$ one can derive that $G$ has strict polynomial growth, i.e. there are a symmetric generating neighbourhood $U$ of $G$ and constants $C_{1}, C_{2}, D>0$ such that $C_{1} k^{D} \leq\left|U^{k}\right| \leq C_{2} k^{D}$ for all $k \in \mathbb{N}$. This allows one to use a result of Hebisch and Saloff-Coste [HS] on pointwise Gaussian estimates for convolution powers of probability densities. We need this for 
Proposition 3. If $\sigma_{L_{\omega}^{1}(G)}(f)=\sigma_{L^{1}(G)}(f)$ for all $f \in L_{\omega}^{1}(G)$, then $\omega$ satisfies the GRScondition and even condition $(S)$.

For the proof of this, one makes use of the above-mentioned estimate for a continuous symmetric probability density with compact support. This yields an inequality also involving an integral over $U^{k}$, where $U$ is a generating neighbourhood. The point now is to estimate this integral from below by the integral over a carefully chosen subset $E_{k}$ of $U^{k}$. The rest then follows by suitable estimates and limit operations.

Sketch of the proof of the theorem:

(iii) $\Leftrightarrow$ (iv): see [FGLLM], Theorem 3.6 and Lemma 3.8.

(iv) $\Rightarrow$ (ii) is Proposition 3 .

(ii) $\Rightarrow$ (i) has been noted after the definition of condition $(S)$.

(i) $\Rightarrow$ (iii): The general line of proof is as in the proof of [FGLLM], Theorem 13. In Lemma 3.12 of [FGLLM] replace the assumption that the weight $\omega$ on $G$ satisfies condition $(S)$ by the weaker one that the quotient weight $\dot{\omega}_{\left.\right|_{H}}$ on $H / N$ satisfies condition $(S)$. Then the proof given there is still valid. In the proof of the theorem, this Lemma is applied repeatedly to pairs of subgroups $G_{i-1} \supset G_{i}$ (the $G_{j}$ coming from Losert's structure theorem [Lo]). In this situation, the quotient weight $\dot{\omega}_{\left.\right|_{G_{i-1}}}$ clearly satisfies the GRScondition, so by Lemma 2, condition $(S)$ is satisfied on $G_{i-1} / G_{i}$. So everything is fine, and (iii) follows.

REMARK 1. A few more equivalent conditions can be added to the theorem (see [FGLLM]). Let us mention just two of them:

(v) $r_{L_{\omega}^{1}(G)}(f)=r_{B(H)}(L f)$ for all $f \in L_{\omega}^{1}(G)$

(vi) $\sigma_{L_{\omega}^{1}(G)}(f)=\sigma_{B(H)}(L f)$ for all $f \in L_{\omega}^{1}(G)$.

Here, $L: f \mapsto L f$ denotes the left regular representation of $L_{\omega}^{1}(G)$ on $H=L^{2}(G)$ and $B(H)$ is the algebra of all bounded linear operators on $H$.

It would be sufficient, to ask (v) and (vi) for selfadjoint elements $f \in L_{\omega}^{1}(G)$ (see [FGLLM]). Condition (v) then reads:

(vii) $r_{L_{\omega}^{1}(G)}(f)=\|L f\|_{B(H)}$ for all $f=f^{*} \in L_{\omega}^{1}(G)$.

\section{References}

[FGL] G. Fendler, K. Gröchenig, and M. Leinert, Symmetry of weighted $L^{1}$-algebras and the GRS-condition, Bull. London Math. Soc., to appear.

[FGLLM] G. Fendler, K. Gröchenig, M. Leinert, J. Ludwig, and C. Molitor-Braun, Weighted group algebras on groups of polynomial growth, Math. Z. 245 (2003), 791-821.

[FRW] J. B. Fountain, R. W. Ramsay, and J. H. Williamson, Functions of measures on compact groups, Proc. R. Ir. Acad. Sect. A 76 (1976), 235-251.

[G] Y. Guivarc'h, Croissance polynomiale et périodes des fonctions harmoniques, Bull. Soc. Math. France 101 (1973), 333-379.

[GL1] K. Gröchenig and M. Leinert, Wiener's lemma for twisted convolution and Gabor frames, J. Amer. Math. Soc. 17 (2004), 1-18. 
[GL2] K. Gröchenig and M. Leinert, Symmetry and inverse-closedness of matrix algebras and functional calculus for infinite matrices, Trans. Amer. Math. Soc. 358 (2006), 2695-2711.

[HS] W. Hebisch and L. Saloff-Coste, Gaussian estimates for Markov chains and random walks on groups, Ann. Prob. 21 (1993), 673-709.

[Lo] V. Losert, On the structure of groups with polynomial growth II, J. London Math. Soc. 63 (2001), 640-654.

[Lu] J. Ludwig, A class of symmetric and a class of Wiener algebras, J. Functional Analysis 31 (1979), 187-194.

[S] T. Strohmer, Four short stories about Toeplitz matrix calculations, Linear Algebra Appl. 343/344 (2002), 321-344.

[SF] S. Shirali and J. W. M. Ford, Symmetry of complex involutory Banach algebras II, Duke Math. J. 37 (1970), 275-280. 